\title{
Characterizing Thermal Runaway in HTS Current Leads
}

\author{
J. M. Pfotenhauer and J. W. Lawrence \\ University of Wisconsin - Madison, Madison, WI, USA
}

\begin{abstract}
Following a brief review of the many issues pertinent to HTS current lead design, we describe the performance of a pair of 1500 A HTS current leads during the transient warm-up following a loss of intercept cooling. Results describing the subsequent temperature evolution are provided both from experimental measurements and from an associated ANSYS model. The study confirms that HTS current leads must be analyzed on a systems basis - incorporating the thermal and electrical characteristics of all components - to provide useful information for the end user. A performance contrast is provided between conduction-cooled and convection-cooled modes. It is found that when heat generation in the HTS conductor exceeds convective cooling by a factor of 10 or more, that part of the current lead is thermally decoupled from the rest of the lead.
\end{abstract}

\section{INTRODUCTION}

At first glance, one might expect that the task of communicating current from room temperature to the cryogenic environment of a superconducting magnet would be a relatively simple design problem, especially as compared to the larger cHullenge of designing a complete superconducting magnet system and its associated cryogenic environment. Furthermore, one might expect that with the significant attention given to vapor-cooled current lead designs prior to 1987 [1] , the addition of HTS elements would be a relatively minor perturbation on an already mature technology. Nevertheless, over the past ten years a surprising variety of issues have been identified and explored in the pursuit of an optimized, low-heat-leak, highly-reliable current lead. These may be roughly categorized according to the three major components in an HTS current lead: the upper (conventional) stage, the thermal intercept, and the lower (HTS) stage.

The issues which have been addressed with respect to the upper stage design are in part a revisit of issues explored in the 1960s. However, with the increased likelihood for vapor-

Manuscript received September 15, 1998.

This work was supported in part by The University of Wisconsin System, and American Superconductor Corp. cooled designs using cryogens other than liquid helium, and the already common use of conduction-cooled designs tied to cryocoolers, an awakened interest has developed for a general analysis addressing upper-stage optimization. The results confirm previous knowledge that the optimum design is defined by an appropriate aspect ratio, that a good heat exchanger design is required for vapor-cooled designs, and that the cold end heat leak is defined purely by the choice of end temperatures and operating current.

The new HTS current leads incorporate a thermal intercept at the junction between the upper and lower stages, and allow for a variety of cooling choices and electrical isolation methods.

Issues which have been addressed for the lower stage include material choice, configurations which will reduce the influence of magnetic field on HTS current carrying capacity, the use of a parallel current shunt for magnet protection, heat load measurements, thermal runaway, the influence of contact resistance between the HTS and conventional materials, and the use of a variable HTS cross sectional area to reduce the cold end heat leak.

Although early motivation for HTS lead development was derived on the basis of reduced helium boiloff, various authors [2-6] have recognized that the broader system design goal is to minimize the room-temperature refrigeration power required to keep the current lead cold.

Among the yet unexplored issues are the possibility of incorporating a position-dependent cross sectional area in upper stage, designing leads for anticipated duty cycles rather than for maximum current operation, optimization based on the load-dependent floating temperature characteristic of cryocoolers, as well as others. In the present work we investigate yet another relatively unexplored issue by combining experimental measurements with a numerical model to describe HTS thermal runaway characteristics.

\section{THERMAL RUNAWAY CHARACTERIZATION}

Experimental efforts on HTS leads at UW-Madison over the past few years have focused on their behavior following loss of cooling at the thermal intercept. The effort has been motivated by the desire to provide the end-user with a definition of the operational limitations of HTS current leads, and to investigate methods for increasing their reliability. A parallel numerical effort has been initiated using the commercial finite-element modeling package ANSYS. This 
approach is being pursued primarily for reasons of flexibility, and in the hope that the method can be easily adopted elsewhere.

\section{A. Experimental description}

Details of the experimental test rig have been given elsewhere [7,8], however for clarity, distinctive characteristics are repeated here. The current leads are designed with an maximum upper stage capacity of $1500 \mathrm{~A}$, intercept cooling provided by a GM cryocooler, and HTS elements fabricated from a stacked tape of BSCCO - 2223 / Ag-Au alloy material with nominal dimensions of $48 \mathrm{~cm}$ length by $0.48 \mathrm{~cm}^{2}$ total cross section. The Ag - $3 \mathrm{wt} \%$ Au alloy comprises $~ 70 \%$ of the HTS composite conductor cross section. Copper bus bars, of $7.2 \mathrm{~kg}$ each, connect the thermal intercept on the current leads to the two voltage-isolated cold fingers of the GM cryocooler. The bus bars provide both a low resistance thermal path between the current leads and cryocooler, and a large thermal mass retarding thermal runaway following loss of intercept cooling.

The experimental procedure is initiated by establishing thermal equilibrium with current flowing in the leads and cooling provided at the cryocooled intercept. The bottom of the leads are shorted with a superconducting cable which is bathed in liquid helium. The transient thermal behavior of interest is caused by shutting off the cryocooler at the thermal intercept. In the data presented below the temperatures, when reported at a fixed location, are as measured on the stacked tape of superconductor $1 \mathrm{~cm}$ down from the intercept-tosuperconductor joint.

\section{B. ANSYS model}

A two-dimensional axi-symmetric model is used to describe the current lead utilizing the PLANE67 thermoelectric element. In addition to spatial configuration, this element type permits one to either define or determine temperature, voltage or electric current, joule-heat generation, density, and material properties at the various nodes. Heat transfer coefficients or heat flux values may be defined at the element faces. Definition of the temperature dependent material properties for thermal conductivity, electrical resistivity, density, heat capacity, and enthalpy are established in look-up tables which ANSYS uses in its calculations. Separate look-up tables for the electrical resistance of the HTS material at each value of current (I) are defined through published critical current $\left(I_{c}\right)$ data [9] and the relationship for the effective resistance, $\mathbf{R}_{\text {eff }}$, of the composite conductor at temperatures above the current sharing temperature, $\mathrm{T}_{\mathrm{cs}}$ :

$$
R_{e f f}=\frac{R_{A g}\left[I-I_{c}(T)\right]^{2}+R_{A g}\left[I-I_{c}(T)\right] I_{c}(T)}{I^{2}}
$$

Here $R_{a g}$ is the resistance of the silver matrix, $I(T)$ is the total current, and $I_{c}$ is the temperature dependent critical current.

The geometrical configuration of the current lead is defined by a grid of 16 vertical by 4 radial nodes for the upper (copper) stage, 4 vertical by 4 radial nodes for the copper bus/thermal intercept, and 16 vertical by 4 radial nodes for the lower (HTS) stage. Boundary conditions are fixed at the vertical ends with $\mathrm{T}_{\text {bottom }}=4.2 \mathrm{~K}$ and $\mathrm{T}_{\text {top }}=300 \mathrm{~K}$, at the thermal intercept through a heat flux which produces the same equilibrium temperature as determined experimentally, and by an adiabatic boundary condition at the axial nodes, and the outer radius nodes of the upper and lower stages. Transient behavior of the lead is initiated by changing the thermal intercept boundary condition from a finite heat flux to an adiabatic condition.

\section{Results and Discussion}

1) Conduction cooled: As presented in a previous publication [8], the temperature rise of the current lead from its equilibrium condition (before turning off the cryocooler) to the point when the upper end of the HTS material reaches $T_{c s}$ is dominated by the thermal capacity of the copper bus bar at the thermal intercept. This behavior is displayed in Fig. 1 as determined experimentally with $\mathrm{I}=1000 \mathrm{~A}$, and with $\dot{m}=0$ (i.e. in the conduction cooled mode), and as calculated with the ANSYS model. The model is shown to very accurately describe the somewhat non-linear temperature rise to $T_{c s}$, followed by the rapid thermal runaway associated with the heat generated in the superconductor.

In view of this success, and in place of subjecting the HTS lead to possible permanent damage, the ANSYS model has been used to determine the thermal runaway behavior up to a peak temperature near $400 \mathrm{~K}$. The results for the same 1000 A conduction cooled case are displayed in Fig. 2. It is

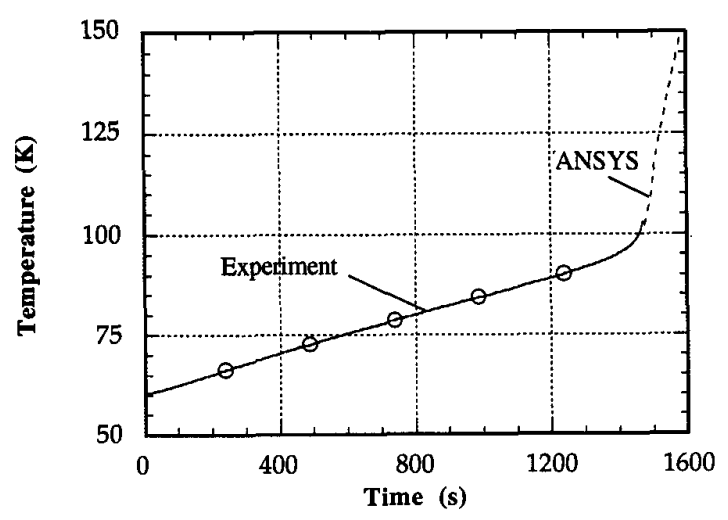

Fig. 1. Comparison of experimental data and ANSYS results for temperature rise following shutdown of intercept cooler. For clarity only a few data points are shown. Solid line $=$ data, dashed line $=$ ANSYS results. 


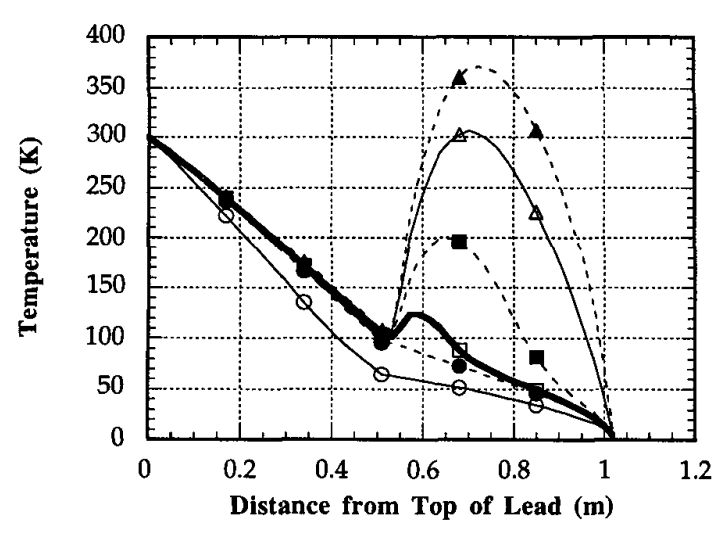

Fig. 2 ANSYS generated description of thermal runaway with $I=1000 \mathrm{~A}$, conduction cooled. $O, t=0$ sec., $Q, t=1250$ sec., $\square, r=1500$ sec., $\square, t=$ 1600 sec., $\Delta, \mathrm{t}=1700 \mathrm{sec}$, $\Delta, \mathrm{t}=1750 \mathrm{sec}$.

interesting to note that the time required (500 seconds) to rise from $T_{c s}$ to $400 \mathrm{~K}$ is in good agreement with the results presented by Heller \& Hull [10]. The same time is lower than predicted by Wesche \& Fuchs [3], but this may be due to the different thermal properties of the $\mathrm{Ag}-\mathrm{Au}$ alloy (this study) and the pure $\mathrm{Ag}$ used in reference [3]. The good agreement with the data from Heller and Hull is not surprising since the current density in the $\mathrm{Ag}-\mathrm{Au}$ alloy during the thermal runaway process is very close to $3 \mathrm{kA} / \mathrm{cm}^{2}$ in both of these studies. However, this agreement reveals the fact that the time dependent thermal runaway in the HTS conductor is a process which is thermally decoupled from the rest of the current lead. Thus the most rapid, and potentially dangerous, thermal behavior in the lead may be calculated from the Ag-Au composite properties alone.

Further confirmation of the decoupled nature of the HTS thermal runaway is shown in figure 3 , where the peak

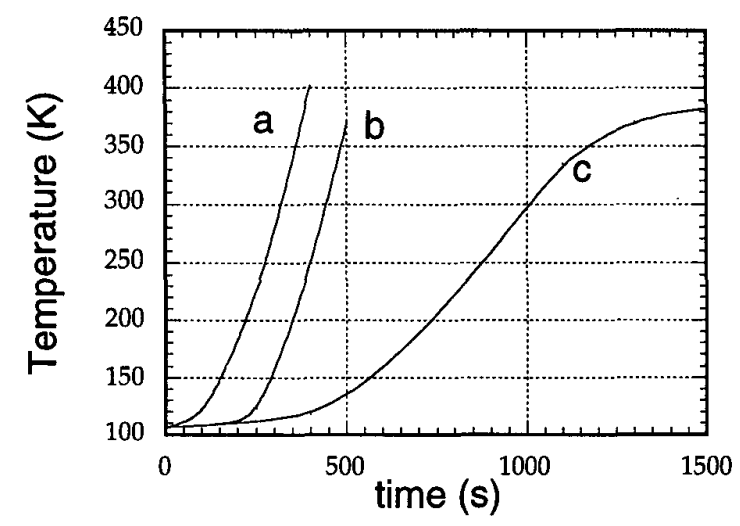

Fig. 3 ANSYS generated peak HTS temperature during thermal runaway for $1000 \mathrm{~A}$. a) without bus bar at thermal intercept. b) with bus bar at thermal intercept. c) with bus bar at thermal intercept and with laminar convective cooling. temperatures, at whatever point they occur within the HTS conductor, are plotted vs. time for the 1000 A operating current. Here $t=0$ is defined by the time at which the HTS element reaches $T_{c s}$. Lines a) and b) compare the cases modeled in ANSYS without and with the massive copper bus bar at the thermal intercept locations. The offset at low temperatures reveals the early influence of the bus bar, but as the temperature increases, both curves display the same slope indicative of the fact that the HTS hot-spot temperature is decoupled from the thermal inertia of the bus bar. Note that the agreement between line a) and the results in Heller \& Hull $[10]$ is essentially exact, as it should be, since their model did not include any significant thermal mass at the intercept.

2) Convective cooling - ANSYS: The third curve displayed in figure 3 (line $\mathrm{c}$ ) results from modifying the ANSYS model to incorporate convective cooling of helium gas up through the lead. Clearly the convective cooling significantly influences the thermal runaway behavior.

Several comments must be made with respect to convective cooling within the ANSYS model, as this is determined through the heat transfer coefficient and fluid temperature at each axial location. First of all, we have chosen to use a constant heat transfer coefficient over the entire length of the current lead. The experimental program has included both self-cooled and forced-flow cooling modes, and even for the highest mass flow rates of $0.1 \mathrm{~g} / \mathrm{s}$, flow through all parts of the lead is laminar. For well known cases of internal laminar flow, the heat transfer coefficient is independent of the mass flow rate; that is, it is constant.

Secondly, our model has approximated the mean fluid temperature as a linearly increasing function with temperature gradients in the lower and upper stages inversely proportional to the thermal conductivities of the solid materials in those regions. This approximation has been justified by the relatively good agreement between the experimental and modeled results shown in figure 4. However, the

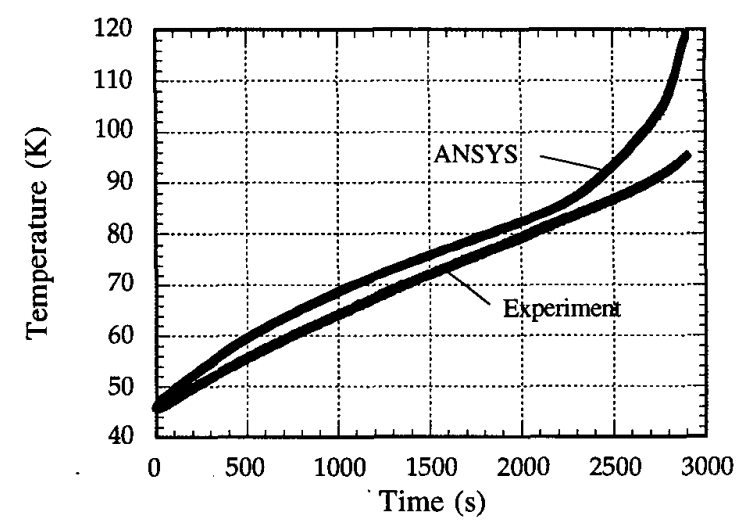

Fig. 4 Comparison of ANSYS and experimental results for temperature rise following shut down of intercept cooler: $1000 \mathrm{~A}$, convection (self) cooled case with mass flow of $0.017 \mathrm{~g} / \mathrm{s}$. 
approximation is sufficiently unsatisfactory upon physical grounds to prohibit use of the model for predictive behavior in the thermal runaway process. Alternative approaches for more accurately characterizing the convective cooling within ANSYS are being pursued.

3) Convective cooling - Empirical observations: A series of tests has been conducted at $1000 \mathrm{~A}$, measuring the transient warming and thermal runaway of the current lead with a forced flow of cold $(4.2 \mathrm{~K})$ helium gas introduced at the bottom of the lead (flowing out the top) at the same time that the intercept cryocooler is turned off. Helium flow rates range from 0 to $0.1 \mathrm{~g} / \mathrm{s}$. A significant increase is seen in the time required for the superconductor to reach $T_{c s}$ - compare for example the time scales in figures 4 and figure 1 - and the thermal runaway process is also greatly retarded. In fact with sufficient cooling (mass flow rates above $0.8 \mathrm{~g} / \mathrm{s}$ ) a superconducting recovery from the thermal runaway is observed. This observation confirms the possibility for an active protection scheme for the HTS leads.

Data from these tests have been combined with results from similar tests under self-cooled conditions at $750 \mathrm{~A}$ and $1250 \mathrm{~A}$. Recognizing that the energy carried away by the gas flow in each case is characterized by the quantity $\dot{m} \mathrm{c}_{\mathrm{P}, \mathrm{f}} \mathrm{dT} / \mathrm{dx}$, we define the dimensionless variable $\alpha$,

$$
\alpha=\frac{J^{2} \rho A_{c}}{\dot{m} c_{p, f}(d T / d x)}
$$

which represents the ratio of heat generated to that carried away by the gas per unit volume of the conductor and cooling channel combined. Here $\mathrm{J}$ is the current density in the Ag-Au alloy, and the temperature gradient is within the fluid. For each experimental test, thermal runaway was halted when the peak temperature in the HTS material was $\sim 105 \mathrm{~K}$. The fluid temperature gradient is then equal to $210 \mathrm{~K} / \mathrm{m}$ for all tests. The ratio of the time required to increase from $\mathrm{T}_{\mathrm{cs}}$ to $\sim 105 \mathrm{~K}$ for the convective-cooled case to that for the conduction-cooled case is plotted against $\alpha$ in figure 5. Here one may observe that the benefit of convective cooling for retarding thermal runaway is realized in a significant degree only when $\alpha<10$. For values of $\alpha<4$ thermal runaway may be avoided altogether. However, even with the self cooled case (here $\alpha=17$ ), one gains a 60\% increase in time.

\section{CONCLUSIONS}

A modeling approach using the commercial finite element package ANSYS has been shown to be especially well suited for modeling the transient behavior of conduction cooled leads. An associated expression is given to characterize the joule heat generation of the composite HTS conductor for temperatures above the current sharing temperature. The ANSYS model confirms the adiabatic nature of the thermal runaway process.

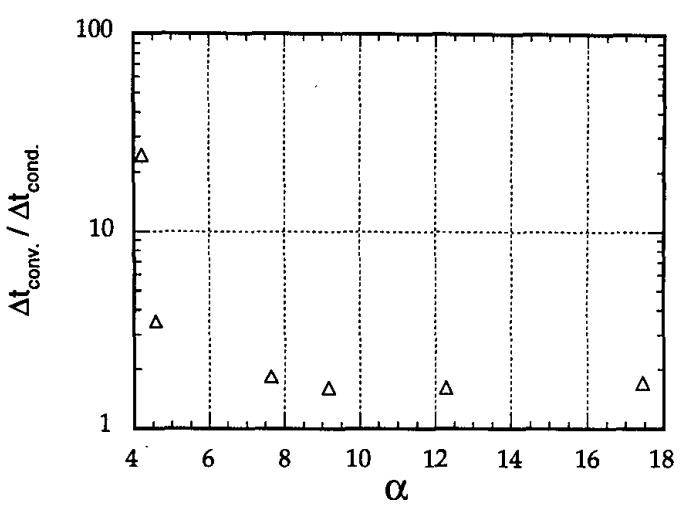

Fig. 5 Thermal runaway rise-time ratio from convection- and conductioncooled data, plotted against dimensionless ratio of joule heating to convective cooling.

Nominal success has been achieved using ANSYS to model convectively cooled leads, however further effort is required in this direction. Experimental data reveal that it is possible, through the addition of sufficient helium mass flow, to provide an active protection mechanism for the HTS leads. Finally, it is empirically demonstrated that the addition of convective cooling is effective in slowing thermal runaway only when the ratio of joule heat production to convective cooling is less than 10 .

\section{REFERENCES}

[1] Yu.L. Buyanov, "Current Leads for use in Cryogenic Devices. Principle of Design and Formulae for Design Calculations," Cryogenics, vol. 25, pp. 94-110, February 1985.

[2] Cryogenics, vol. 25, pp. 94-110, February 1985. Verhaege, C. Albrecht, and J. Bock, "Cryogenic Load Calculation of High Tc Current Lead," Cryogenics, vol. 33, pp. 555-562, March 1993.

[3] R. Wesche and A.M. Fuchs, "Design of Superconducting Current Leads," Cryogenics, vol. 34, pp. 145-154, February 1994.

[4] S. Yang and J.M. Pfotenhauer, "Optimization of the Intercept Temperature for High Temperature Superconducting Current Lead," Advances in Cryogenic Engineering vol. 41A, pp. 567$572,1996$.

[5] H.M. Chang and S.W. Van Sciver, "Thermodynamic Optimization of Conduction Cooled HTS Current Leads," to be published in Cryogenics.

[6] C.M. Rey, "Re-evaluation of the Intercept Temperature for HTS Current Leads Based on Practical and Economical Considerations," presented at this conference.

[7] J.M. Pfotenhauer, O.D. Lokken, and P.E. Gifford, "Performance of a Twin Cold Finger Gifford McMahon Cryocooler," in Proceedings of the 16th International Cryogenic Engineering Conference T. Haruyama, T Mitsui, and K. Yamafuji, eds. Elsevier Science, 1997 , pp. 363-366.

[8] J.M. Pfotenhauer and O.D. Lokken, "Test Results of a 1.5 kA HTS Current Lead for $\mu$ SMES," IEEE Trans. on Appl. Current Lead for $\mu$ SMES,
Superconductivity, vol. 7 (2), pp. 363-366, 1997.

[9] R.C. Niemann, D.J. Evans, B.L. Fischer, W.E. Brockenborough, P.R. Roberts, and A.J. Rodenbush, "Characterization of High Current, High-Temperature Superconductor Current Lead Elements," IEEE Trans. on Appl. Superconductivity, vol. 7 (2), pp. 1829-1832, 1997

[10] R. Heller and J.R. Hull, "Conceptual Design of a 20-kA Current Lead using Forced-Flow Cooling and Ag-Alloy-Sheathed Bi-2223 High Temperature Superconductors," IEEE Trans. on Appl. Superconductivity, vol. 5 (2), pp. 797-800, 1995. 